\title{
Adding Epidemiologically Important Meteorological Data to Peanut Rx, the Risk Assessment Framework for Spotted Wilt of Peanut
}

\author{
Thomas M. Chappell, ${ }^{1, \dagger}$ Clarence B. Codod,2 Blake W. Williams, ${ }^{2}$ Robert C. Kemerait, ${ }^{2}$ Albert K. Culbreath, ${ }^{2}$ and \\ George G. Kennedy ${ }^{3}$ \\ ${ }^{1}$ Department of Plant Pathology and Microbiology, Texas A\&M University, College Station, TX 77843, U.S.A. \\ ${ }^{2}$ Department of Plant Pathology, College of Agricultural and Environmental Science, University of Georgia, Tifton, GA 31793, U.S.A. \\ ${ }^{3}$ Department of Entomology and Plant Pathology, College of Agriculture and Life Sciences, North Carolina State University, Raleigh, NC \\ 27695-7630, U.S.A. \\ Accepted for publication 2 March 2020.
}

\begin{abstract}
Management of disease affecting peanut in the southeastern United States has benefited from extensive field research identifying diseaseassociated risk factors since the 1990s. An assessment of risk factors associated with tomato spotted wilt (TSW), caused by tomato spotted wilt virus and spread exclusively by thrips, is available to growers through Peanut Rx, a tool developed to inform peanut management decisions. Peanut Rx provides an assessment of relative TSW risk as an index. The assessment provides information about the relative degree to which a field characterized by a specified suite of practices is at risk of crop loss caused by TSW. Loss results when infection occurs, and infection rates are determined, in part, by factors outside a grower's control, primarily the abundance of dispersing, viruliferous thrips. In this study, we incorporated meteorological variables useful for predicting thrips dispersal,
\end{abstract}

ABSTRACT increasing the robustness of the Peanut Rx framework in relation to variation in the weather. We used data from field experiments and a large grower survey to estimate the relationships between weather and TSW risk mediated by thrips vectors, and developed an addition to Peanut Rx that proved informative and easy to implement. The expected temporal occurrence of major thrips flights, as a function of heat and precipitation, was translated into the existing risk-point system of Peanut Rx. Results from the grower survey further demonstrated the validity of Peanut Rx for guiding growers' decisions to minimize risk of TSW.

Keywords: Arachis hypogaea, disease control and pest management, disease exposure, disease forecasting, disease risk, ecology and epidemiology, Peanut Rx, thrips, tomato spotted wilt virus
Tomato spotted wilt virus (genus Orthotospovirus; family Bunyaviridae) (TSWV), the causal agent of tomato spotted wilt (TSW) in peanut, is transmitted exclusively by thrips (Thysanoptera: Thripidae) and is capable of infecting over 1,000 plant species, causing economic loss to many crops (Culbreath and Srinivasan 2011; Pappu et al. 2009; Ullman et al. 2002; Whitfield et al. 2005). To reduce economic loss due to TSW, a variety of countermeasures has been identified and implemented, including resistant crop cultivars (Branch 2003, 2007a,b; Holbrook and Culbreath 2008; Holbrook et al. 2008; Shrestha et al. 2013), applications of pesticides targeting the thrips vectors or the disease (Culbreath et al. 2016; Wells et al. 2002a,b), and numerous production practices (Culbreath et al. 2013; Hurt et al. 2006; Marois and Wright 2003). Each of these measures reduces TSW risk for a crop by altering the abundance or behavior of the thrips vector, thereby reducing virus transmission, or by reducing susceptibility of the crop to the pathogen (Culbreath et al. 2013; Sundaraj et al. 2014).

The peanut-thrips-TSWV system has been reasonably wellcharacterized epidemiologically. In the United States, peanut was first observed affected by TSW in 1971 in Texas (Philley et al. 1972)

\footnotetext{
${ }^{\dagger}$ Corresponding author: T. M. Chappell; thomas_chappell@tamu.edu
}

Funding: Support was provided by the United States Department of Agriculture (USDA) National Institute of Food and Agriculture Risk Avoidance and Mitigation Program Grant Number RF330-411-3843858 for research leading to basic thrips and tomato spotted wilt virus risk models that formed the foundation of our analysis and USDA National Institute of Food and Agriculture Coordinated Agricultural Project Grant Number 2012-68004-20166 for research and development of the modified model and the analyses reported here.

The author(s) declare no conflict of interest.

(C) 2020 The American Phytopathological Society and was subsequently found in other southeastern states where peanut crops are grown (Black et al. 1986; Culbreath et al. 1992; Hagan et al. 1990; Halliwell and Philley 1974; Reed and Sukamto 1995). TSWV is persistent and propagative (Whitfield et al. 2005) in tobacco thrips (Frankliniella fusca Hinds) (Culbreath et al. 2003; Riley et al. 2011), which is the principal vector in the southeastern United States. The mechanisms of primary spread of TSWV to peanut are similar to those characterizing other crops: $F$. fusca adults transmit TSWV as they immigrate to peanut from diverse host plants in surrounding landscapes (Groves et al. 2001, 2002; Srinivasan et al. 2014), including when alternative host plants senesce due to changing weather or human action (Chappell et al. 2013; Groves et al. 2001). The frequency of viruliferous populations of adult dispersing $F$. fusca is a function of the probability of a thrips encountering a TSWV-infected noncrop host in the seasons leading up to cropping (Chappell et al. 2013), and this probability depends on the abundance of TSWV in alternative hosts in the surrounding areas. Thus, the epidemiology of TSW on peanut is affected by the complex temporal dynamics of TSWV in agricultural landscapes and their surroundings.

Knowledge about TSW in peanut has been effectively translated into the TSW risk assessment framework (Brown et al. 2005), now widely implemented as Peanut Rx (University of Georgia 2018). Peanut Rx can be easily used in the field, and immediately provides a TSW-risk outlook for a crop. The tool is a composite decision aid that treats various peanut production practices and pest and disease management inputs as risk-mitigation factors influencing TSW risk. Peanut Rx allows users to compare the expected reduction in exposure to TSW associated with various TSW management tactics, and provides a weighted index as an estimate of the relative reduction in TSW risk (Brown et al. 2005). The index values are based on results from numerous field studies that estimated the average reduction in TSW incidence associated with each risk 
mitigation practice (e.g., peanut cultivar, tillage, planting date, plant density and row spacing, and herbicide and insecticide use) (Brown et al. 2005; Culbreath et al. 2013; Hurt et al. 2006; Marois and Wright 2003). For any given suite of risk mitigation practices used by a grower, Peanut Rx sums the index values associated with each practice to provide an overall estimate of risk.

Peanut Rx has been widely used since its development. It has been continually updated and improved as new peanut cultivars have become available, as cultivation practices have changed, and as pest- and disease-management products have entered and left the market. Further opportunities for improvement of Peanut Rx exist due to two recent technological developments: first, the increasing quality and availability of real-time meteorological data, and second, the increasing ease with which quantitative models can be operationalized as software. To date, Peanut Rx has implicitly included weather information through the use of calendar-based assignments of risk points in relation to planting date (University of Georgia 2018). Calendar dates allow Peanut Rx to incorporate the relationship between risk of TSW and planting dates under average weather conditions. However, when weather is atypical, TSW incidence can be atypically high or low. In this study, we add realtime weather data to Peanut Rx to take into account the interyear variability in weather as it affects TSW. The anecdotal "late thrips" problem faced by peanut growers is an example of risk variation that can be addressed by incorporating real-time data into Peanut Rx: typically, peak thrips dispersal occurs before peanut crop susceptibility peaks but weather-related delays in thrips population development and dispersal can result in higher than average risk to crops.

Predicting the seasonal phenology of TSWV's thrips vectors in other cropping systems has been successful. A predictive model that forecasts potential TSW incidence in tobacco is based on $F$. fusca phenology (Chappell et al. 2013), and has been implemented as an online tool (North Carolina State Climate Office 2014). A decision aid that equips growers and managers with information and predictions regarding population dynamics and dispersal of western flower thrips ( $F$. occidentalis), the principal vector of TSWV in California, is based on observed weather conditions and has been implemented as a TSWV field risk index tool for tomato production in California's Central Valley (University of California 2019). The ability to predict the abundance of $F$. fusca is useful in the southeastern United States because there is a positive association between disease risk and vector abundance and, when very high numbers of infectious $F$. fusca are present, the efficacy of riskmitigating actions can be reduced or completely negated (Chappell and Kennedy 2018). Therefore, it is important to consider the timing of dispersal and changes in the magnitude of populations of $F$. fusca in relation to the suite of TSW mitigation practices used on the crop.

Tobacco thrips population growth and dispersal from overwintering hosts of TSWV (Chamberlin et al. 1992; Groves et al. 2002) are largely dependent on temperature and precipitation, which are primary drivers of thrips development and dispersal (Morsello and Kennedy 2009; Morsello et al. 2010). Heat drives physiological development of both the thrips and its host plants, while precipitation acts as a major mortality factor for juvenile thrips, suppresses dispersal by adults, and delays senescence of the winter annual plants that serve as overwintering hosts for both $F$. fusca and TSWV. These effects of weather on thrips phenology are included in the "Thrips Infestation Predictor (TIP) for Cotton," a model describing thrips infestation of cotton seedlings (Kennedy et al. 2017), which predicts timing of thrips population development and dispersal as a function of heat and precipitation (the model also takes into account planting date, plant growth, and thrips phenology).

Thus, weather data can be used to predict thrips abundance and dispersal and, by extension, the transmission of TSWV. The agroecosystems of the southeastern United States are diverse but include plant species that serve as overwintering hosts for both
TSWV and F. fusca, providing sources for early-season spread of the virus into susceptible crops (Groves et al. 2001, 2002; Srinivasan et al. 2014). Weather-driven thrips phenology models (Chappell et al. 2013; Kennedy et al. 2017) predict factors relating to transmission risk but do not directly account for variation in risk that derives from growers' actions to suppress TSW. The Peanut Rx framework includes a detailed accounting of production and management actions that affect TSW risk but does not account for variation in transmission risk due to thrips phenology affected by the weather. Thus, including a weather-based component to determine transmission risk of TSWV in Peanut Rx brings mutually complementary sources of information together to improve prediction of TSW incidence in peanut.

\section{MATERIALS AND METHODS}

Field trial dataset. Data were from field trials conducted in Georgia at six research stations of the University of Georgia. The research stations were the Attapulgus Research and Education Center (Attapulgus), the C. M. Stripling Irrigation Research Park (Stripling), the Southwest Georgia Research and Education Center (Plains), the Black Shank Farm (Tifton), the Southeast Georgia Research and Education Center (Midville), and the Vidalia Onion and Vegetable Research Center (Reidsville). The field trials were designed to generate variation in factors described by Peanut $\mathrm{Rx}$, and all had the same split-split plot design. The whole-plot treatments consisted of three planting dates (early, middle, and late), the subplot treatments included two cultivars of peanut, and the subsubplots were treatments with or without phorate (Thimet-20G, AMVAC Chemicals; Newport Beach, CA, U.S.A.) insecticide $(5.6 \mathrm{~kg} / \mathrm{ha}$ in-furrow at planting). Each trial was replicated four times at each of the six locations in each of 2 years (2017 and 2018). One change was made in 2018: in 2017, the cultivars Georgia-06G (Branch 2007a) and FloRun 157 were planted but, in 2018, due to lack of seed, TUFRunner 511 (Tillman and Gorbet 2017) was planted in place of FloRun 157. The cultivar Georgia-06G has field resistance to TSWV while FloRun 157 and TUFRunner 511 are more susceptible. Thus, the trial structure was 2 years by three planting dates by six locations by two cultivars by two insecticide treatments and was replicated four times, giving 576 individual TSW incidence observations at the sub-subplot level. The incidence of TSW was assessed using a "hit-stick method" as described by Culbreath et al. (1997). The number of $0.3-\mathrm{m}$ portions of a row containing symptomatic plants showing concentric ring spots, various patterns of chlorosis, and stunting was counted and converted to a percentage of row length. The row length varied depending on the size of the field in each location, with 15.2-m rows in Tifton, Attapulgus, Plains, and Reidsville; $18.3-\mathrm{m}$ rows in Midville; and 24.4-m rows in Stripling (corresponding to 50, 60, and 80 feet, respectively). Skips (missing plants within a plot) due to seedling mortality from other diseases, planter equipment failure, or damage from pivot irrigation tracks were discounted from the total row length. These trials investigated the effects of 12 different combinations of two peanut cultivars, three planting dates, and application with or without phorate insecticide on TSW incidence. Incidence was quantified based on symptom expression at the end of the field trials on 1, 13, and 15 September for Attapulgus/Stripling, Midville/Reidsville, and Plains/Tifton locations, respectively. These dates correspond to a range of 98 to 133 days after planting. At these locations, the three planting dates ranged from 15 to 30 April, 10 to 25 May, and 26 May to 10 June, respectively.

Survey dataset. A survey was conducted across the peanut production region of Georgia during 2017 and 2018 to assess the levels of risk and TSW incidence in commercial peanut fields. In 2017, the survey was conducted from 25 July to 3 October and, in 2018, it was conducted between 2 August and 24 September. Using a stratified sampling approach, 20 counties distributed across the peanut production region were selected. Fields were randomly 
selected from each county, with more fields being selected from counties with greater area devoted to peanut production. In all, 73 commercial peanut fields in 20 counties were surveyed in 2017 and 83 fields in 21 counties were surveyed in 2018. In each field, four $15.2-\mathrm{m}$ plots were arbitrarily established within each field and flagged for repeated ratings. Areas in the field exhibiting nematode infestation, flooded conditions, weedy zones, and areas beyond irrigation were avoided because such conditions would interfere with TSW assessment. Two rows per plot (total of $30.5 \mathrm{~m}$ ) were rated for TSW incidence in 2017. In 2018, four rows per plot were assessed; thus, the total row length per plot was $61.0 \mathrm{~m}$. The survey dataset was collected primarily to assess the performance of Peanut $\mathrm{Rx}$ in the current production environment. In total, 624 observations were assembled in the survey dataset.

Using these data, we examined how accounting for weather effects on thrips populations could be integrated into the existing Peanut Rx. Where possible, we used variables and factors that are present in the existing Peanut Rx framework (University of Georgia 2018). For example, a given peanut cultivar is assigned a number of risk points for the "cultivar" factor that reflected the level of TSW resistance for that cultivar, with TSW-susceptible cultivars receiving more points (University of Georgia 2018). The total points make up the risk summary provided by Peanut Rx as it is used in practice. We included each risk category (source of risk points) as an independent variable in models describing TSW incidence; the level
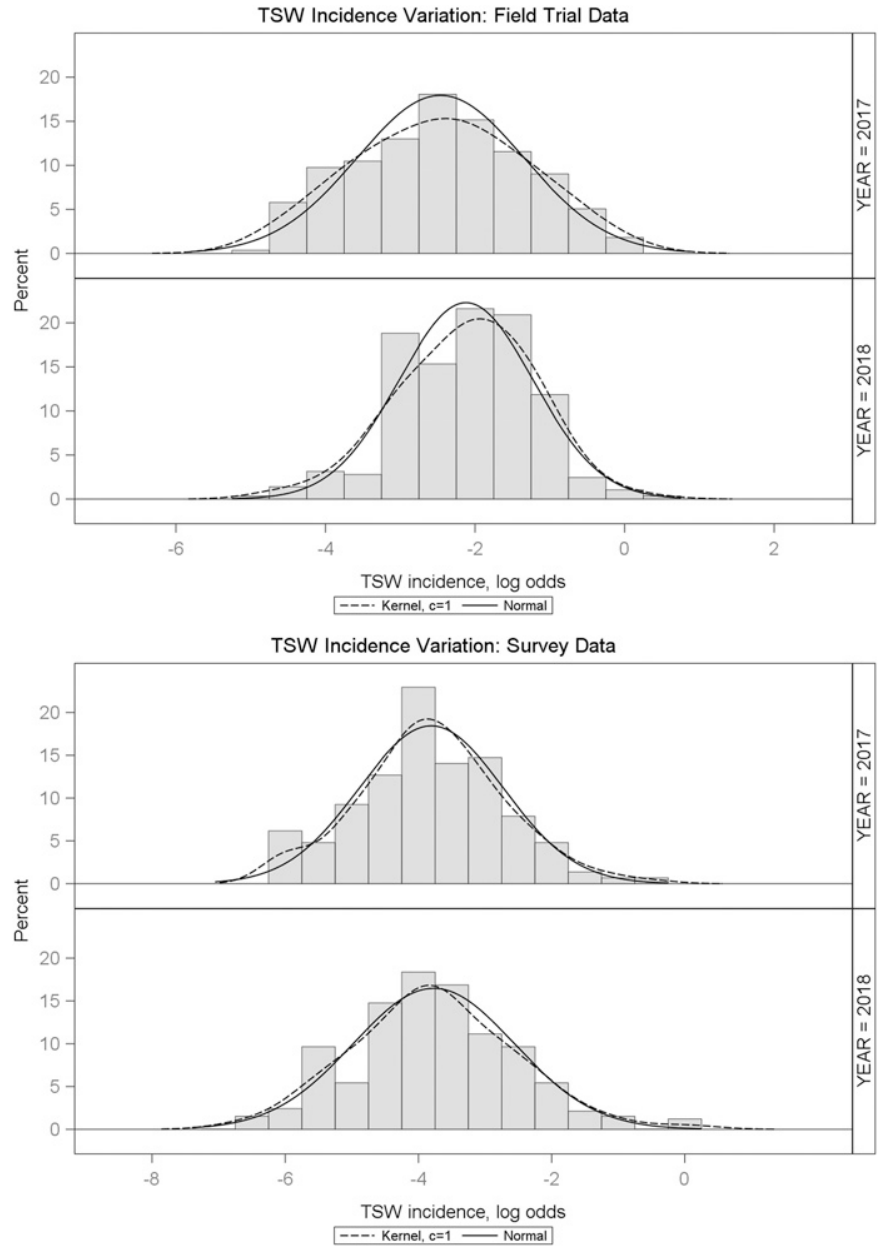

Fig. 1. Tomato spotted wilt (TSW) incidence variation in field trial and survey datasets. Histograms are shown for each of the 2 years (2017 and 2018), with TSW incidence quantified as log odds of infection. Each histogram pertaining to the field trial dataset reflects 288 incidence observations. Histograms pertaining to the survey dataset reflect 292 and 332 observations for 2017 and 2018, respectively. of each risk category generally varies in multiples of 5 risk points, depending on the specific case in Peanut Rx (University of Georgia 2018). For example, a peanut cultivar associated with relatively high TSW risk may be assigned 15 risk points, whereas a cultivar with lower TSW risk would be assigned 5 risk points. Updated Peanut Rx publications provide details concerning the distribution of risk points.

Naturally occurring variation in weather between locations and years was analyzed to identify relationships between weather variables and TSW incidence. We used output from the TIP model (Kennedy et al. 2017) of thrips phenology, specifically the predicted time at which the highest number of adults from the fourth annual generation of F. fusca should be dispersing, as a basis for deriving candidate variables to represent what we call "transmission intensity." We consider transmission intensity to be the aggregate result of multiple processes, including $F$. fusca development, dispersal, feeding, and frequency of viruliferous individuals in the dispersing vector population. Currently, candidate variables are not interpreted to provide information about the rate of viruliferousness but are expected to represent development, dispersal, and feeding through correlation with thrips population dynamics. Essentially, we expect a positive correlation between relative abundance of dispersing thrips and relative transmission intensity, though we note that the ability of weather-based variables to contribute to Peanut Rx output does not strictly depend on this interpretation. Derived variables analyzed in this study included crop age at the time of
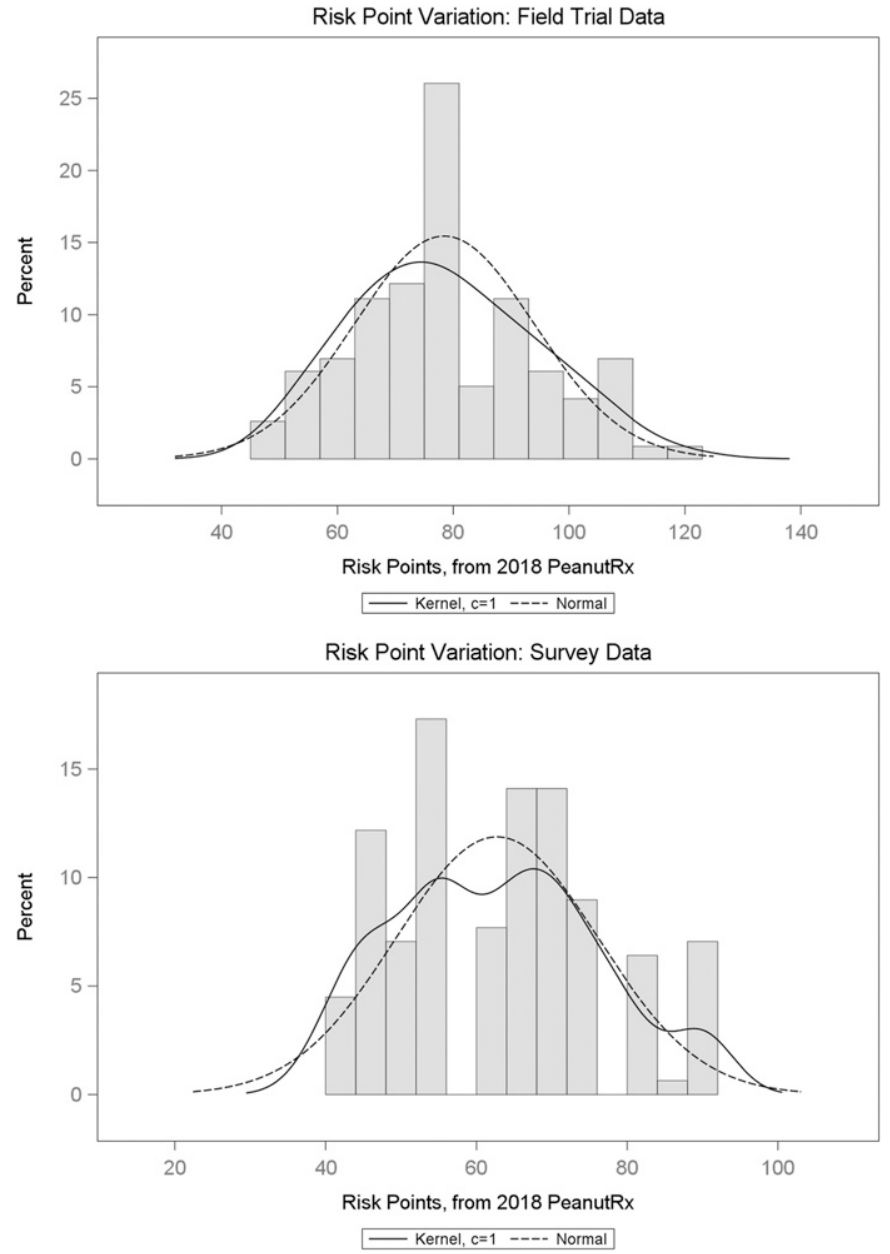

Fig. 2. Peanut Rx (2008 version) tomato spotted wilt (TSW) risk point variation for field trial and survey datasets, shown as one histogram for all 576 field trial observations and one histogram for all 624 survey observations. Risk point totals for each observation are calculated as the sum of risk points from all Peanut Rx point categories (e.g., cultivar and tillage), and are assigned at the plot level for the field trial data and the field level for the survey data-one plot or field has one TSW risk value represented by Peanut Rx risk points. 
maximum expected thrips dispersal (the difference, in days, between peanut planting date and expected fourth-generation thrips dispersal); the difference between current-year maximum thrips dispersal time and a 5-year average of thrips dispersal times relativized to the given location based on its average, which provides an indication of seasonal lateness or earliness of thrips dispersal; and a simple categorization of thrips dispersal timing as being early, middle, or late. We included thrips dispersal in our study because the TSWV or thrips mitigation decisions that Peanut $\mathrm{Rx}$ incorporates in its projection of risk depend on an assumption that TSWV is being transmitted to the peanut crop. We expected that TSW risk associated with planting date would be conditioned upon the time at which thrips disperse and transmit TSWV in the given year. For example, what is typically an optimal planting date within the 10-to-31 May window (Brown et al. 1996; Brown et al. 2003), chosen in part to avoid thrips dispersal, could become a high-risk planting date if thrips dispersal timing deviates appreciably from its typical pattern. Because this risk-avoidance practice of optimizing the time of planting is the tactic most widely adopted by growers (Srinivasan et al. 2017), the importance of planting time to reduce TSW risk is likely well understood by growers.

All estimates of thrips dispersal timing generated by the TIP are based on weather (Kennedy et al. 2017). The North Carolina State Climate Office's Climate Retrieval and Observations Network of the Southeast (CRONOS) database includes, at the time of this publication, records from 38,407 weather-recording sites
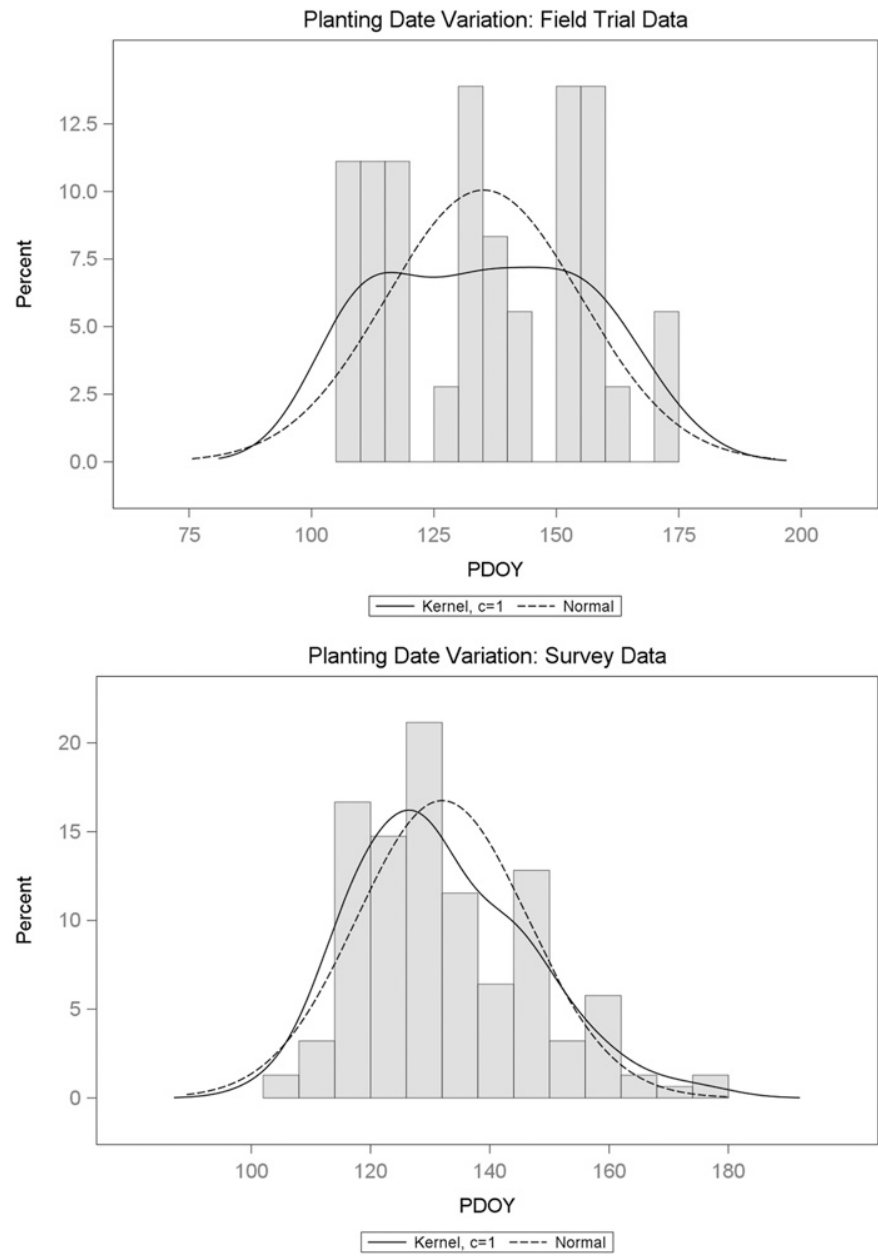

Fig. 3. Planting date variation for field trial and survey datasets. Comparison of histograms shows that, in the field trial, there was appreciably more planting date variation than in the survey data, because growers more consistently plant crops during times associated with reduced risk of Tomato spotted wilt. PDOY $=$ planting day of year. nationally. Historical precipitation and temperature data were collected from the CRONOS database for use in estimating timing of thrips dispersal using the TIP (Kennedy et al. 2017). These data include hourly temperature observations beginning each year at a thrips "biofix" date of 15 November. This date corresponds with the only $F$. fusca dispersal event that occurs in the fall and is associated with thrips movement from summer to winter plant hosts. Heat accumulations based on a lower threshold of $10.5^{\circ} \mathrm{C}$ (Lowry et al. 1992) were derived from temperature records in the CRONOS database and used to estimate calendar dates of thrips dispersal for each of four adult generations, each separated by 237 degree days. More than four thrips generations occur in a given year but only the four occurring during the peanut growing season were considered here; further, the temporal occurrence of all thrips generations is ordered and autoregressive, such that the timing of the fourth generation is a useful indicator of the timing of all generations. Precipitation records in the CRONOS database are processed to output a standardized precipitation index (McRoberts and NielsenGammon 2012), the value of which is negatively correlated with thrips population development.
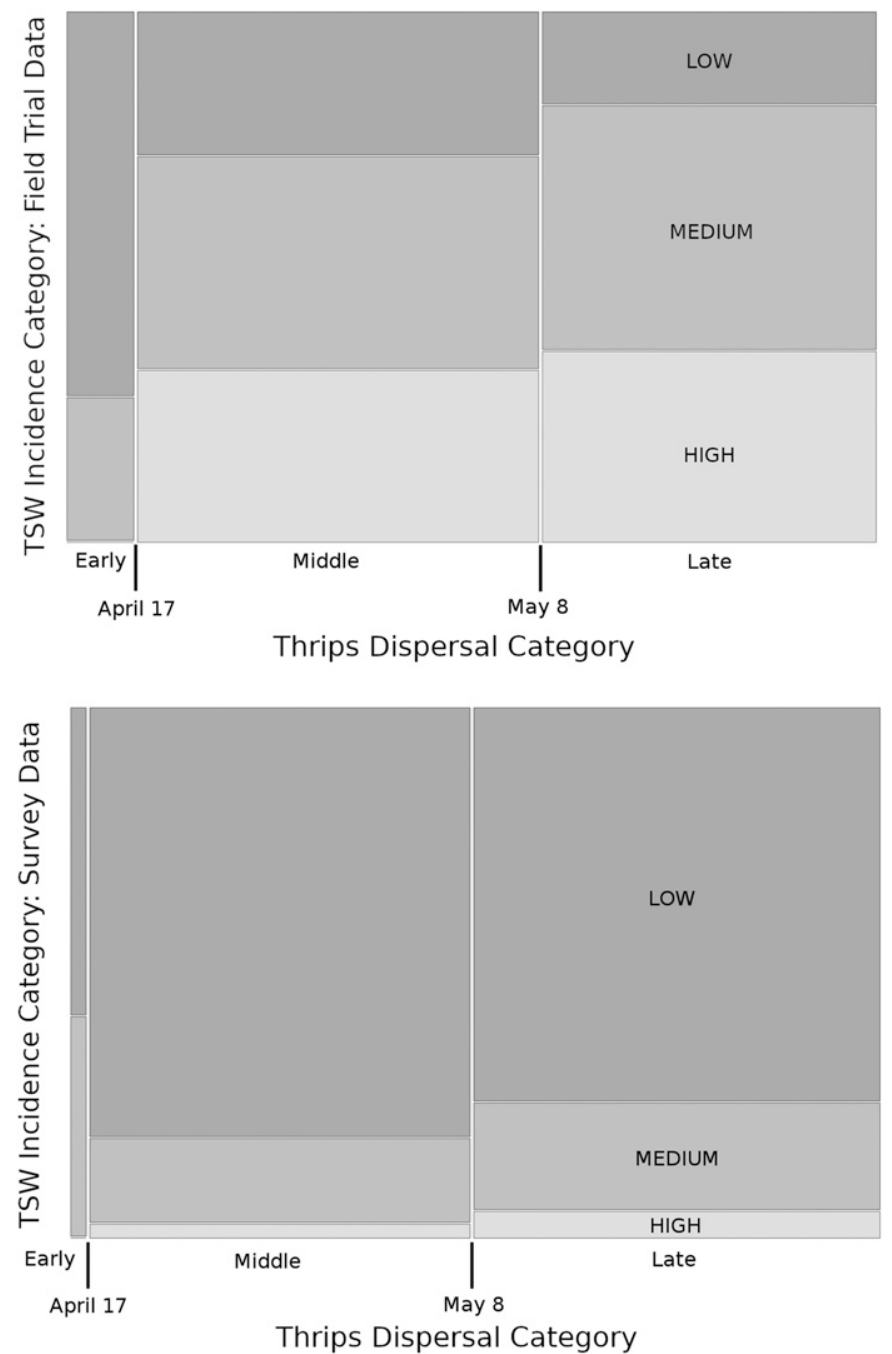

Fig. 4. Frequency plot of Tomato spotted wilt (TSW) incidence category (low, medium, or high) by thrips dispersal category (early, middle, or late) for field trial and survey datasets. TSW incidence is categorized as low if it is $0 \%$ up to and including 5\%, medium if it is greater than 5\% and less than or equal to $15 \%$, and high if it is greater than $15 \%$. Area of the rectangular sections in the plot correspond to the relative frequency of observations in the given combinations of TSW incidence and thrips dispersal categories. Comparison between field trial and survey data shows that, in general, risk points assigned to grower fields are lower than those assigned to experimental scenarios generated through field trials. 
Statistical analysis. We categorized field trial and survey incidence records to correspond with occurrence of incidence within prescribed ranges, consistent with how Peanut Rx is used to assign risk categories of high, medium, and low as a function of cumulative risk points. We used a categorization of three incidence levels based on quartile limits $(\mathrm{Q} 1=4.7 \%$ and Q3 $=17.5 \%)$ from our field trial dataset: a low category of $0 \%$ up to and including $5 \%$ incidence, a middle category greater than $5 \%$ up to and including $15 \%$ incidence, and a high category of greater than $15 \%$ incidence. For comparing performance of the contemporary version of Peanut Rx (hereafter "benchmark") (University of Georgia 2018) with candidate incorporations of thrips-related data, we used a two-level categorization of TSW incidence (a low and high category). We varied the threshold between these two categories, using each of 5.0, 7.5 , and $10 \%$ as boundaries, where incidence values below or equal to the threshold are categorized as low. We also used median percent incidence as a threshold.

We sought to incorporate information into an existing quantitative understanding of the peanut-thrips-TSWV system. Because Peanut Rx outputs risk assessment as a sum of risk points, we proposed the addition of points to represent risk related to thrips phenology: "thrips risk points" are assigned based on weather data and a model-based interpretation of weather data that results in an estimate of adult thrips dispersal timing. In short, the TIP uses temperature and precipitation data to calculate an adult thrips dispersal probability distribution, which is a composite of distributions corresponding to thrips generations. The maximum dispersal probability is associated with the fourth adult generation of thrips, and the date on which this maximum occurs was used as a reference for describing the timing of thrips dispersal within a season. Consistent with studies of $F$. fusca phenology in the field (Morsello and Kennedy 2009; Morsello et al. 2010), seasonally higher temperatures result in earlier thrips dispersal, and wetter seasons delay the time at which peak dispersal occurs.

We categorized thrips dispersal timing into three classes: early, middle, and late. The basis for this simplified categorization is the interval during which the fourth adult generation of thrips is expected to be dispersing. The dates of the threshold between early and middle intervals and the threshold between the middle and late intervals were based on quantile limits, as in the categorization of TSW incidence described earlier. We estimated peak $F$. fusca dispersal dates for each location-year combination represented in the aggregated field trial dataset and, from the distribution of these dates, estimated quartile limits. The early threshold was established as 17 April (Q1) and the late threshold as 8 May (Q3). We used these dates to categorize thrips dispersal timing as follows: if fourthgeneration dispersal was estimated to be between the thresholds, dispersal was categorized as middle. Otherwise, dispersal was early if before the early threshold and late if after the late threshold. Points associated with thrips dispersal categories were estimated by fitting a generalized linear model of incidence variation, including thrips dispersal category as an independent variable. The GLIMMIX procedure of SAS (SAS V9.4) (SAS Institute Inc. 2015) was used for analysis and included two input variables as fixed effects: Peanut Rx (University of Georgia 2018) risk points and thrips category. For the field trial dataset, experimental design variables were included as random effects: plot (spatial block) and year. For the survey dataset, survey design variables were included as random effects: county (spatial block) and year. In each case, covariance was structured as variance components. TSW incidence as a proportion was modeled as the dependent variable, assuming a $\beta$ response distribution and logit link function. Coefficients estimated for the three thrips categories were interpreted into point assignments, similar to the practice of deriving point assignments from field study results of other factors that make up Peanut Rx's index.

Results of adding thrips-related information to Peanut $\mathrm{Rx}$ were investigated by quantifying classification performance. The GLIMMIX procedure was used to estimate relationships between risk point totals for Peanut Rx variables and binary TSW incidence category for both the survey and trial datasets. Risk point assignments to field trial and survey data were made on the basis of the 2018 University of Georgia Peanut Disease Risk Index (University of Georgia 2018), in which point assignments for peanut cultivars, tillage, and other management practices may be found, with their rationale and experimental basis explained in detail. Receiver operating characteristic (ROC) curve analysis was used to assess classification performance. The area under the ROC curve (AUROC), $c$, was calculated and used to compare classification performance. For three-level categorizations, frequency tables were generated to show associations between thrips timing categories and TSW incidence categories.

Confusion matrices (Pearson 1904) were generated to demonstrate the consequences of varying the threshold (potentially based on economic injury or other levels) used to classify TSW incidence. Confusion matrices show the performance of a risk-point-driven classifier with chosen classification thresholds by tabulating frequencies of true and false positives and true and false negatives. Because growers are variably risk averse, demonstrating the consequences of setting a lower or higher incidence threshold as a basis for decision making (an "action threshold", which can vary independently of an economic injury or other threshold) shows how false-positive or negative rates change commensurately. The 90th percentile boundary was $9 \%$ for TSW incidence in the survey dataset (i.e., $90 \%$ of incidence observations lie below 9\%). We used this boundary as one basis for interpreting model performance, showing how growers could rely on the Peanut Rx tool to correctly tell them whether their incidence would be among the highest decile. We also varied thresholds for both true and predicted occurrences to show how variable risk aversion need not defeat the ability of the Peanut $\mathrm{Rx}$ forecasting framework to provide quantitative output for decision support. For example, to represent the decision-making process of a grower seeking an unbiased estimate (one equally likely to be high or low relative to reality), we used the same threshold for classifying predictions and observations. In this case, observed TSW incidence above threshold was classified as high and predictions above the same threshold were classified as high. This approach distributes error equally above and below estimates, resulting in unbiased output. In a further example, and to represent the decision-making process of a risk-averse grower (one who deploys management at a lower action threshold based on prediction than the same grower would deploy if he or she knew the future accurately), we classified observed TSW incidence using one threshold but classified predictions using a lower threshold. In this case, the threshold for classifying incidence observations might be $10 \%$ but the threshold for classifying high versus low risk might be at $7 \%$ predicted incidence. Consequences of separating these thresholds are obvious in general: increasing risk aversion results in fewer false negatives and more false positives or, in other words, fewer misses and more deployment of effort not necessary in hindsight. However, quantitatively, the consequences of varying risk aversion are not obvious. To address this, we varied thresholds, fit logistic regression models of TSW incidence category as a function of Peanut Rx risk points calculated using the benchmark (University of Georgia 2018) or modified scheme, summarized classifier performance, and present results that demonstrate how false-positive and false-negative rates change, using the survey dataset as a representation of real-world conditions in the target cropping system.

\section{RESULTS}

TSW incidence and risk point variation. The field trial dataset showed appreciable variation in TSW incidence, ranging from 0 to $59.6 \%$ TSW symptomatic row-feet of assessed peanut. This variation, computed as log odds of infection, was normally distributed (Fig. 1). Although the range of TSW incidence 
denominated as a percentage exceeded 50\%, median TSW incidence was $9.4 \%$. Here, we address the difference in variability above and below median incidence through use of a threshold that harmonizes analysis output with its applied purpose of supporting binary decisions concerning TSW management. The survey dataset also showed appreciable variation in TSW incidence; however, it was apparent that TSW incidence reported in the surveys was lower than that observed in the field trials. Again, TSW incidence variation, computed as $\log$ odds of infection, was normally distributed (Fig. 1). Survey-reported TSW incidence ranged from 0 to $53.1 \%$, with a median value of $2.1 \%$ - a contrast with the median of $9.4 \%$ for the field trial dataset, and more than a fold change in average odds of infection between the field and survey datasets.

Variation in total risk points in the field trial dataset varied around a mean of 78.4 (standard deviation $[\mathrm{SD}]=15.5$ ), while variation in total risk points in the survey dataset varied around a mean of 62.7 $(\mathrm{SD}=13.4)$, being 15.7 risk points lower than the field trial dataset, on average (Fig. 2). Planting date variation in the field trial dataset was structured as a function of the experimental design, ranging from 16 April to 22 June (Fig. 3). Quartile-based thrips date thresholds were established on 18 April (108th day of year) and 8 May (128th day of year). Planting dates reported in the survey dataset were more clustered around the average of 12 May than were planting dates in the field trial dataset.

The frequency of TSW incidence categories and thrips dispersal timing categories as proportional areas can be represented graphically (Fig. 4). Using the survey dataset to fit a model of TSW incidence, estimated coefficients for thrips risk categories were $0.159,0$, and 0.207 for early, middle (the reference level), and late, respectively. The coefficient in the same model relating Peanut $\mathrm{Rx}$ risk points to TSW incidence was 0.025 . With these parameter estimates as a guide, we assigned thrips points to dispersal timing categories as follows: 10 for early thrips dispersal, 0 for middle, and 15 for late. We rounded these point assignments for operational compatibility with the existing Peanut Rx. Whereas the field trial represented variation that included many high-risk configurations by design, the survey dataset included fewer high-risk configurations and commensurately fewer high-incidence occurrences. The general relationship between thrips timing category and TSW incidence category was consistent in results for the field trial and survey datasets: in general, higher incidence, though rare, occurs more frequently when thrips disperse late.

TABLE 1. Classification of sub-subplots from the field trial dataset based on set Tomato spotted wilt (TSW) incidence thresholds for benchmark (Peanut Rx) (University of Georgia 2018) and modified (including thrips dispersal points) risk point formulas

\begin{tabular}{lccccc}
\hline & \multicolumn{2}{c}{ Number } & & \multicolumn{2}{c}{$c$ (AUROC) $^{\mathrm{a}}$} \\
\cline { 2 - 3 } \cline { 5 - 6 } TSW incidence threshold $(\%)$ & Below & Above & & Benchmark & Modified \\
\hline 5.0 & 166 & 410 & & 0.627 & 0.719 \\
7.5 & 229 & 347 & & 0.609 & 0.703 \\
10.0 & 304 & 272 & & 0.618 & 0.712 \\
Median (9.4) & 288 & 288 & & 0.615 & 0.815 \\
\hline
\end{tabular}

a $c($ AUROC $)=$ area under the receiver operating characteristic curve.

TABLE 2. Classification of fields described in the survey dataset based on set Tomato spotted wilt (TSW) incidence thresholds for benchmark (Peanut Rx) (University of Georgia 2018) and modified (including thrips dispersal points) risk point formulas

\begin{tabular}{lcccccc}
\hline & \multicolumn{2}{c}{ Number } & & \multicolumn{2}{c}{$c\left(\right.$ AUROC) $^{\mathrm{a}}$} \\
\cline { 2 - 3 } \cline { 6 - 6 } TSW incidence threshold (\%) & Below & Above & & Benchmark & Modified \\
\hline 5.0 & 487 & 137 & & 0.686 & 0.703 \\
7.5 & 541 & 83 & & 0.738 & 0.772 \\
10.0 & 574 & 50 & & 0.748 & 0.792 \\
Median (2.1) & 312 & 312 & & 0.649 & 0.651 \\
\hline
\end{tabular}

a $c($ AUROC $)=$ area under the receiver operating characteristic curve.
A summary of classification results for models fit to the trial dataset is presented in Table 1, in which TSW incidence category is binarized on the basis of the indicated threshold (below or including, or above), and modeled as a function of Peanut Rx risk points (benchmark model) (University of Georgia 2018) or as a function of Peanut Rx risk points to which thrips dispersal points have been added (modified model, this study). It can be seen that classification performance is improved; for example, from an AUROC of 0.615 for the benchmark model to an AUROC of 0.815 for the modified model, when classification of incidence is made based on the median of $9.4 \%$.

Summary results of logistic regression models fit to the survey dataset are presented in Table 2, in which TSW incidence category is binarized on the basis of the indicated threshold (below or including, or above), and modeled as a function of benchmark Peanut Rx risk points (University of Georgia 2018) or as a function of Peanut Rx risk points to which thrips points have been added (modified model, this study). Noteworthy is the increasing benefit of including thrips-related information as the TSW incidence threshold increases. Thrips information is more important for classifying high or low incidence at a $10 \%$ threshold than it is for a lower threshold whereas, at very low thresholds such as the survey dataset's median $2.1 \%$, thrips-related information adds little to the description of incidence as a classification.

ROC curves overlaid for benchmark and modified models are presented and have different area under the curves: $\chi^{2}=7.504, P=$ 0.0062 (Fig. 5; Table 2).

Validation of Peanut Rx using the survey dataset. The survey dataset collected from commercial peanut growers provides unprecedented opportunity to validate Peanut Rx. It also provides opportunity to communicate to participating and other growers some of the practical consequences of making risk management decisions based on optimistic versus pessimistic expectations.

Confusion matrices for incidence classifications (Table 3) show classification performance of logistic regression models based on

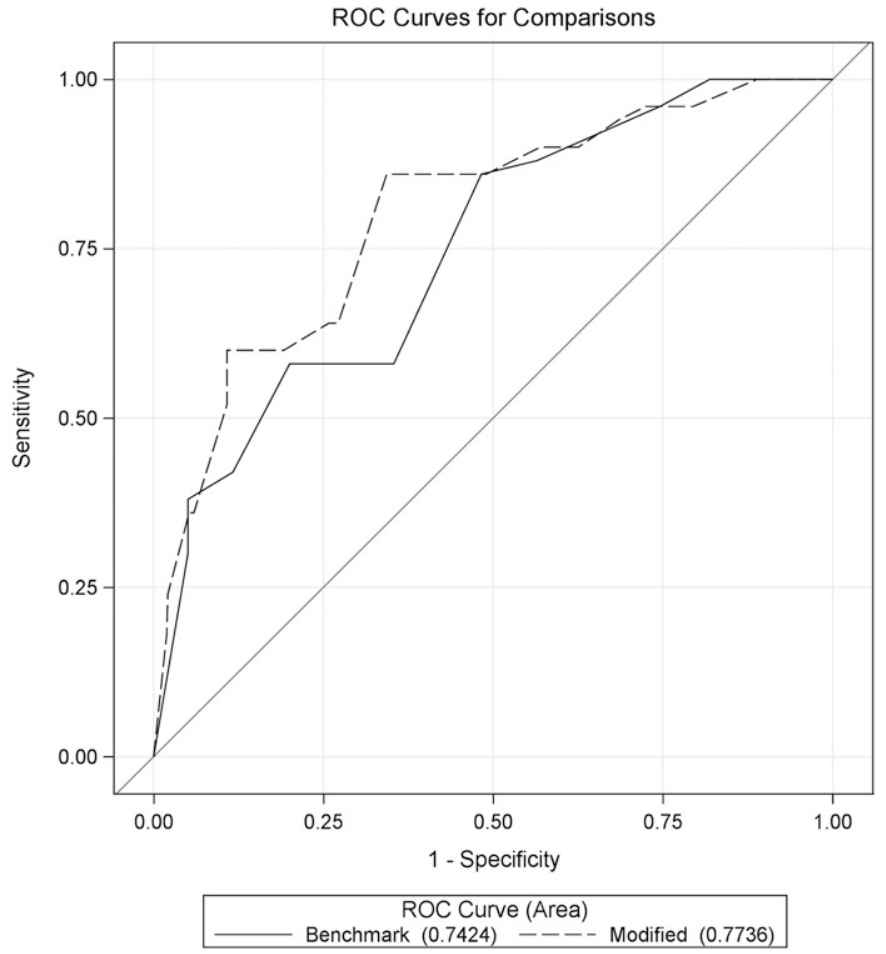

Fig. 5. Receiver operator characteristic (ROC) curves for benchmark (Peanut Rx) (University of Georgia 2018) and modified (including thrips dispersal points) models, using the $90 \%$ quantile boundary of $9 \%$ incidence of Tomato spotted wilt as a classification threshold. Area under the ROC curve contrast: $\chi^{2}=7.504, P=0.0062$. 
the benchmark (University of Georgia 2018) and modified risk point distributions for models fit to the survey data. The 90th percentile boundary of $9 \%$ was used in this analysis. The number of false negatives is halved (27 to 13) as a result of adding thrips dispersal points to the model, while the number of false positives rate is not detrimentally affected-indeed, the false-positive rate is slightly reduced (197 to 175).

Confusion matrices for incidence classifications can be used to show the effects of changing the classification scheme (Table 4). Here, we used a $10 \%$ incidence threshold for classifying observed high versus low and a $7 \%$ incidence threshold for classifying predicted high versus low. The consequence is increased sensitivity at the expense of specificity: a higher true-positive rate but also a higher false-positive rate. This is intentional bias toward the less costly failure, which we suggest is equivalent to risk aversion.

Classification performance, calculated as rates, shows the improvements that result from adding thrips dispersal points to Peanut Rx (Table 5). Here, positive and negative are interpreted to mean incidence values above and below threshold, respectively. These rates include accuracy (number of true classifications divided by the total number of observations), precision (number of true positives divided by the number of predicted positives), specificity (number of true negatives divided by the number of predicted negatives), and sensitivity (number of true positives divided by the number of predicted positives). Specificity can also be calculated as 1 - the false-positive rate and sensitivity as 1 - the false-negative rate. Improvement in sensitivity is appreciable (from 0.565 to 0.790 ), without reducing specificity (it is improved from 0.649 to 0.689). To achieve an improvement of this magnitude without increasing the false-positive rate is possible only because the thripsrelated information included in the modified model is relevant, and absent from the benchmark Peanut Rx model. High-risk situations are associated with the thrips risk points based on timing of dispersal of adult $F$. fusca, and these points are rarely assigned to situations that end up being realized as low risk.

\section{DISCUSSION}

Introducing the Peanut Rx risk assessment framework as it currently exists, Brown et al. (2005) wrote:

\begin{abstract}
"Success in this endeavor can be measured in terms of improved $\mathrm{R}^{2}$ values of plotted survey data, but with a preference of overestimating, rather than underestimating, incidence. In other words, we strive to minimize the number of outlying points on the upper left-hand corner [in which underestimates of high incidence would be found] of our incidence versus index value plots, thereby reducing the number of fields having more disease than the index would predict."
\end{abstract}

In keeping with this preference, we have used a newly collected survey dataset to study how inclusion of weather-based tobacco thrips dispersal projections in the Peanut Rx framework alters risk

TABLE 3. Tomato spotted wilt (TSW) incidence classifications for benchmark (Peanut Rx) (University of Georgia 2018) and modified (including thrips dispersal points) logistic regression models for the survey dataset, with classification threshold of $9 \%$ incidence for both predicted and observed TSW incidence $^{\mathrm{a}}$

\begin{tabular}{lccc}
\hline & & \multicolumn{2}{c}{ Observed TSW category } \\
\cline { 3 - 4 } Model & Predicted TSW category & High, $>9 \%$ & Low, $\leq 9 \%$ \\
\hline Benchmark & High, $>9 \%$ & 35 & 197 \\
& Low, $\leq 9 \%$ & 27 & 365 \\
Modified & High, $>9 \%$ & 49 & 175 \\
& Low, $\leq 9 \%$ & 13 & 387 \\
\hline
\end{tabular}

a Thresholds: observed $=9 \%$ and predicted $=9 \%$. projections within the realistic setting of commercial peanut production, and to assess whether doing so offers potential to improve Peanut Rx. We provide Peanut Rx users with some ability to understand how the intensity of any user's preference for overestimating incidence affects management decisions by presenting Peanut Rx output both as risk points and as a correspondence of risk-point-based predictions to real TSW incidence observations. The most important result of this study is that it builds on the goals set by Brown et al. (2005) and increases Peanut Rx's performance in identifying high-risk situations that arise due to weather variation, thereby identifying situations that would, without the weather data, represent fields "having more disease than the index would predict."

The result of adding relevant weather data to the Peanut $\mathrm{Rx}$ framework is that sensitivity to true positives is increased without increasing the false-positive rate. This makes Peanut Rx more useful for growers who have low tolerance for TSW and false-negative predictions. As indicated by the earlier quotation from Brown et al. (2005), Peanut Rx was designed from the beginning to have a minimal false-negative rate, biasing projections away from the costlier of two potential failures (crop loss versus potentially unnecessary risk mitigation). This approach is appropriate given that the goal is to minimize risk and unnecessary management inputs, and the enhancements reported here increase both components of Brown and colleagues' stated goal.

Whereas Peanut Rx without weather data correctly classifies $56 \%$ of the high-risk ( $>9 \%$ incidence, the upper decile) instances corresponding to inputs from the survey dataset, Peanut Rx with weather data correctly classifies $79 \%$ of these instances. Increasing the true-positive rate of any classifier can be achieved without changing its input data by simply biasing the classifier, but at a cost of generating a commensurately increased number of false positives. The benefit of adding weather data to Peanut $\mathrm{Rx}$ is apparent in a comparison of the false-positive rate for the benchmark and modified models: without weather data, there are 197 false positives among the 624 total data points; adding the weather data reduces the number to 175 . Both represent high falsepositive rates but this is reasonably expected from a framework purposefully biased toward favorable outcomes in the context of uncertainty. Through adding weather data to account for risk that results from variable thrips dispersal timing, the threshold for classifying predictions as high risk can thus be lower without resulting in as many false negatives as would be expected if the

TABLE 4. Tomato spotted wilt (TSW) incidence classifications for benchmark (Peanut Rx) (University of Georgia 2018) and modified (including thrips dispersal points) logistic regression models for the survey dataset, with classification threshold of $7 \%$ incidence of TSW for predicted incidence (action threshold) but $10 \%$ threshold for observed TSW incidence ${ }^{\mathrm{a}}$

\begin{tabular}{lccc}
\hline & & \multicolumn{2}{c}{ Observed TSW category } \\
\cline { 3 - 4 } Model & Predicted TSW category & High, $>10 \%$ & Low, $\leq 10 \%$ \\
\hline Benchmark & High, $>7 \%$ & 29 & 203 \\
& Low, $\leq 7 \%$ & 21 & 371 \\
Modified & High, $>7 \%$ & 40 & 184 \\
& Low, $\leq 7 \%$ & 10 & 390 \\
\hline
\end{tabular}

a Thresholds: observed $=10 \%$ and predicted $=7 \%$.

TABLE 5. Classification performance differences between benchmark (Peanut Rx) (University of Georgia 2018) and modified (including thrips dispersal points) models using the $90 \%$ quantile boundary of $9 \%$ incidence of tomato spotted wilt as the classification threshold

\begin{tabular}{lcccc}
\hline Model & Accuracy & Precision & Specificity & Sensitivity \\
\hline Benchmark & 0.641 & 0.056 & 0.649 & 0.565 \\
Modified & 0.699 & 0.079 & 0.689 & 0.790 \\
\hline
\end{tabular}


threshold were decreased without commensurately improving the classifier's performance by adding information.

The TSW incidence categorization we used is expedient in providing growers of varying risk aversion with actionable information by associating risk point totals with incidence outcomes in a way that prioritizes prediction of high or low incidence. The use of categories allows quantification or classification to focus on variation that is targeted for prediction or description. Assigning incidence or risk point values to categories propagates assumptions about the importance of category limits and within-category variation. Our assumptions were related to downstream applications. The rationale for this categorization is that categories describe variation relevant to management decisionmaking. Incidence variation occurs when incidence is above $15 \%$ but it is probably not important to management decisionmaking - if incidence is above this high threshold, then available management actions are likely to be taken whether incidence is $15 \%$ or appreciably greater than $15 \%$. By matching the interpretation of risk realization to the situations that Peanut $\mathrm{Rx}$ is designed to forecast, we believe this approach can maintain Peanut Rx's historical success in balancing complexity of the peanut-thripsTSWV system against the practical necessity of operability.

We assigned incidence values to categories, collapsing quantitative variation into a limited number of levels for an applied reason: Peanut Rx output is not used to predict expected incidence per se; instead, it is used to inform TSW management decisions. A beneficial consequence of this mode of usage is that different growers, with varying tolerances for TSW incidence, can all use Peanut Rx effectively because Peanut Rx relativizes risk. This is underscored by the fact that Peanut Rx outputs risk points and not expected TSW incidence. Points translate to expected TSW incidence, but not uniformly for all locations, times, or growers; however, the rank-order relationship of risk points to TSW incidence is robust and consistent.

A simple and powerful conclusion can be drawn from the survey dataset: growers are making peanut management decisions that minimize TSW risk. Peanut Rx has been a conduit of information to growers concerning how and why to reduce TSW risk. Peanut Rx has also incorporated additional risk assessment of other disease challenges to peanut, supporting growers' system-level management decisions that are not naive concerning potential issues with overlap in management logistics.

The generalities and error in our results demonstrate that information derived empirically from studies of thrips can improve TSW incidence predictions. This indicates that there are aspects of F. fusca biology that are not only important to the spread of TSWV in peanut but also predictable to some degree. More research is needed. When testing the efficacy of TSW management measures, data on dispersal of vector populations could be directly related to efficacy at an observed transmission risk, which can lead to improved understanding of the generality of the results. For example, a management tool may decrease TSW risk by reducing the frequency with which an individual thrips probes and feeds on a plant, thereby reducing the probability of virus transmission (Jacobson and Kennedy 2013). However, this can be negated if there is a large-enough increase in the number of infectious thrips that challenge the treated plants, thereby eroding the treatment's effectiveness in reducing virus spread (Chappell and Kennedy 2018). Whereas a trial that does not account for variation in vector activities will recover varying efficacy results depending on the unknown transmission risk at trial time, a trial that accounts for transmission risk will be able to cast efficacy in a more informative and realistic context. Although conducting many trials and averaging treatment efficacy over the variation in risk is, in principle, an alternative, this alternative cannot provide insight into functional relationships between treatment efficacy and risk value, for reasons similar to the difficulty in inferring functional meanvariance relationships using averages. To gain that insight, relevant aspects of risk must be observed or credibly projected. The abundance of available data and the existing thorough understanding of management practices' affecting both thrips and TSW incidence makes the Peanut Rx framework a noteworthy area in which to gain that insight.

A critical component affecting TSW incidence in any TSWVthrips cropping system is the abundance of TSWV in the inoculum sources from which it is transmitted to crops. Previous studies (Chappell et al. 2013) have suggested a relationship between abundance of TSWV reservoirs and prior-year weather. This relationship is interpreted as a composite effect of weather on prioryear thrips activity that influences the abundance of TSWV in the landscape the following spring, and is based on a combination of limited TSW incidence data in noncrop sentinel plants and the finding that prior-year weather is consistently associated with TSW incidence in crops. Efficacy trials do not typically account for TSW incidence rates in natural reservoirs. For any thrips' reservoir-tocrop transit, the probability that the reservoir plant from which the thrips has emigrated is infected informs the probability that the thrips is viruliferous; consequently, the effect of thrips population density on transmission hazard is scaled by the incidence of TSW in the reservoir landscape. With adequate sampling providing incidence of TSW in the reservoir landscape available at the time of a trial, alongside observation of thrips-mediated transmission, efficacy trials would be more powerful in recovering meaningful estimates of treatment efficacy. For polyphagous vectors, sampling for TSW incidence in the landscape would need to include more host plant species than would similar sampling in systems with greater host specificity of vectors or viruses.

Once these components are well understood for other TSWVthrips-crop systems, future studies of the type reported here will be able to recover robust composite frameworks of pathosystem dynamics, offering researchers improved understanding of empirical studies, and offering stakeholders improved accuracy in risk assessment. Indeed, Peanut Rx stands as an example of a successful and widely adopted risk assessment framework.

\section{ACKNOWLEDGMENTS}

We thank two anonymous reviewers and the editor for their expertise and input, which led to improvement of this manuscript.

\section{LITERATURE CITED}

Black, M. C., Lummus, P. F., Smith, D. H., and Demski, J. W. 1986. An epidemic of spotted wilt disease in south Texas peanuts in 1985. Proc. Am. Peanut Res. Ed. Soc. 18:66.

Branch, W. D. 2003. Registration of 'Georgia-02C' peanut. Crop Sci. 43: 1883-1884.

Branch, W. D. 2007a. Registration of 'Georgia-06G' peanut. J. Plant Regist. 1:120.

Branch, W. D. 2007b. Registration of 'Georgia Greener' peanut. J. Plant Regist. 1:121.

Brown, S. L., Culbreath, A. K., Todd, J. W., Gorbet, D. W., Baldwin, J. A., and Beasley, J. P., Jr. 2005. Development of a method of risk assessment to facilitate integrated management of spotted wilt of peanut. Plant Dis. 89:348-356.

Brown, S. L., Todd, J. W., and Culbreath, A. K. 1996. Effect of selected cultural practices on incidence of tomato spotted wilt virus and populations of thrips vectors in peanuts. Acta Hortic. 431:491-498.

Brown, S., Todd, J., Culbreath, A., Baldwin, J., Beasley, J., Kemerait, B., Prostko, E., and Smith, N. 2003. Minimizing spotted wilt of peanut. Univ. Georgia, Coop. Ext. Serv. Bull. 1165:12.

Chamberlin, J. R., Todd, J. W., Beshear, R. J., Culbreath, A. K., and Demski, J. W. 1992. Overwintering hosts and wingform of thrips, Frankliniella spp., in Georgia (Thysanoptera: Thripidae): Implications for management of spotted wilt disease. Environ. Entomol. 21:121-128.

Chappell, T. M., Beaudoin, A. L., and Kennedy, G. G. 2013. Interacting virus abundance and transmission intensity underlie tomato spotted wilt virus incidence: An example weather-based model for cultivated tobacco. PLoS One 8:e73321

Chappell, T. M., and Kennedy, G. G. 2018. Estimating the effectiveness of imidacloprid when used to suppress transmission of tomato spotted wilt 
orthotospovirus in commercial agriculture. J. Econ. Entomol. 111: 2024-2031.

Culbreath, A. K., Selph, A. C., Williams, B. W., Kemerait, R. C., Jr., Srinivasan, R., Abney, M. R., Tillman, B. L., Holbrook, C. C., and Branch, W. D. 2016. Effects of new field resistant cultivars and in-furrow applications of phorate insecticide on tomato spotted wilt of peanut. Crop Prot. 81:70-75.

Culbreath, A. K., and Srinivasan, R. 2011. Epidemiology of spotted wilt disease of peanut caused by Tomato spotted wilt virus in the southeastern U.S. Virus Res. 155:101-109.

Culbreath, A. K., Todd, J. W., and Brown, S. L. 2003. Epidemiology and management of tomato spotted wilt in peanut. Annu. Rev. Phytopathol. 41: 53-75.

Culbreath, A. K., Todd, J. W., Demski, J. W., and Chamberlin, J. R. 1992. Disease progress of spotted wilt in peanut cultivars Florunner and Southern Runner. Phytopathology 82:766-771.

Culbreath, A. K., Todd, J. W., Gorbet, D. W., Shokes, F. M., and Pappu, H. R. 1997. Field response of new peanut cultivar UF 91108 to tomato spotted wilt virus. Plant Dis. 81:1410-1415.

Culbreath, A. K., Tubbs, R. S., Tillman, B. L., Beasley, J. P., Jr., Branch, W. D., Holbrook, C. C., Smith, A. R., and Smith, N. B. 2013. Effects of seeding rate and cultivar on tomato spotted wilt of peanut. Crop Prot. 53:118-124.

Groves, R. L., Walgenbach, J. F., Moyer, J. W., and Kennedy, G. G. 2001. Overwintering of Frankliniella fusca (Thysanoptera: Thripidae) on winter annual weeds infected with Tomato spotted wilt virus and patterns of virus movement between susceptible weed hosts. Phytopathology 91:891-899.

Groves, R. L., Walgenbach, J. F., Moyer, J. W., and Kennedy, G. G. 2002. The role of weed hosts and tobacco thrips, Frankliniella fusca, in the epidemiology of Tomato spotted wilt virus. Plant Dis. 86:573-582.

Hagan, A. K., Weeks, J. R., French, J. C., Gudauskas, R. T., Mullen, J. M., Gazaway, W. S., and Shelby, R. 1990. Tomato spotted wilt virus in peanut in Alabama. Plant Dis. 74:615.

Halliwell, R. S., and Philley, G. 1974. Spotted wilt of peanut in Texas. Plant Dis. Rep. 58:23-25.

Holbrook, C. C., and Culbreath, A. K. 2008. Registration of 'Georganic' peanut. J. Plant Regist. 2:17.

Holbrook, C. C., Timper, P., Culbreath, A. K., and Kvien, C. K. 2008. Registration of 'Tifguard' peanut. J. Plant Regist. 2:92-94.

Hurt, C. A., Brandenburg, R. L., Jordan, D. L., Royals, B. M., and Johnson, P. D. 2006. Interactions of tillage with management practices designed to minimize tomato spotted wilt of peanut (Arachis hypogaea L.). Peanut Sci. 33:83-89.

Jacobson, A. L., and Kennedy, G. G. 2013. Effect of cyantraniliprole on feeding behavior and virus transmission of Frankliniella fusca and Frankliniella occidentalis (Thysanoptera: Thripidae) on Capsicum annuum. Crop Prot. 54:251-258.

Kennedy, G. G., Chappell, T. M., Ward, R. V., and DePolt, K. T. 2017. Thrips Infestation Predictor for Cotton. http://climate.ncsu.edu/cottonTIP

Lowry, V. K., Smith, J. W., and Mitchell, F. L. 1992. Life-fertility tables for Frankliniella fusca (Hinds) and F. occidentalis (Pergande) (Thysanoptera: Thripidae) on peanut. Ann. Entomol. Soc. Am. 85:744-754.

Marois, J. J., and Wright, D. L. 2003. Effect of tillage system, phorate, and cultivar on tomato spotted wilt of peanut. Agron. J. 95:386-389.

McRoberts, D. B., and Nielsen-Gammon, J. W. 2012. The use of a highresolution standardized precipitation index for drought monitoring and assessment. J. Appl. Meteorol. Climatol. 51:68-83.

Morsello, S. C., Beaudoin, A. L. P., Groves, R. L., Nault, B. A., and Kennedy, G. G. 2010. The influence of temperature and precipitation on spring dispersal of Frankliniella fusca changes as the season progresses. Entomol. Exp. Appl. 134:260-271.
Morsello, S. C., and Kennedy, G. G. 2009. Spring temperature and precipitation affect tobacco thrips, Frankliniella fusca, population growth and Tomato spotted wilt virus spread within patches of the winter annual weed Stellaria media. Entomol. Exp. Appl. 130:138-148.

North Carolina State Climate Office. 2014. Tobacco Thrips Flight and TSWV Intensity Predictor. http://climate.ncsu.edu/thrips

Pappu, H. R., Jones, R. A. C., and Jain, R. K. 2009. Global status of Tospovirus epidemics in diverse cropping systems: Successes achieved and challenges ahead. Virus Res. 141:219-236.

Pearson, K. 1904. Mathematical contributions to the theory of evolution, XIII: On the theory of contingency and its relation to association and normal correlation. Pages 34-35 in: Drapers' Company Research Memoirs, Biometric Series. Cambridge University Press, Cambridge, UK.

Philley, G., Halliwell, R. S., and Horne, C. W. 1972. Tomato spotted wilt virus disease of peanuts. J. Am. Peanut Res. Educ. Assoc. Inc. 5:198.

Reed, J. T., and Sukamto, S. 1995. Thrips and Tomato spotted wilt virus in a Mississippi peanut field. Pages 171-173 in: Thrips Biology and Management. Springer, Boston, MA, U.S.A.

Riley, D. G., Joseph, S. V., Srinivasan, R., and Diffie, S. 2011. Thrips vectors of tospoviruses. J. Integr. Pest Manage. 2:1-10.

SAS Institute Inc. 2015. Base SAS ${ }^{\circledR} 9.4$ Procedures Guide, 5th Ed. SAS Institute Inc., Cary, NC, U.S.A.

Shrestha, A., Srinivasan, R., Riley, D. G., and Culbreath, A. K. 2013. Second generation peanut genotypes resistant to thrips-transmitted Tomato spotted wilt virus exhibit tolerance rather than true resistance and differentially affect thrips fitness. J. Econ. Entomol. 106:587-596.

Srinivasan, R., Abney, M. R., Culbreath, A. K., Kemerait, R. C., Tubbs, R.S., Monfort, W. S., and Pappu, H. R. 2017. Three decades of managing tomato spotted wilt virus in peanut in southeastern United States. Virus Res. 241: 203-212.

Srinivasan, R., Riley, D. G., Diffe, S., Shrestha, A., and Culbreath, A. K. 2014. Winter weeds as inoculum sources of tomato spotted wilt virus and as reservoirs for its vector, Frankliniella fusca (Thysanoptera: Thripidae) in farmscapes of Georgia. Environ. Entomol. 43:410-420.

Sundaraj, S., Srinivasan, R., Culbreath, A. K., Riley, D. G., and Pappu, H. R. 2014. Host plant resistance against Tomato spotted wilt virus in peanut (Arachis hypogaea) and its impact on susceptibility to the virus, virus population genetics, and vector feeding behavior and survival. Phytopathology 104:202-210.

Tillman, B. L., and Gorbet, D. W. 2017. Registration of 'TUFRunner 511' peanut. J. Plant Regist. 11:235-239.

Ullman, D. E., Meideros, R., Campbell, L. R., Whitfield, A. E., Sherwood, J. L., and German, T. L. 2002. Thrips as vectors of tospoviruses. Adv. Bot. Res. 36:113-140.

University of California. 2019. TSWV Field Risk Index and Thrips Projections. University of California Division of Agriculture and Natural Resources. https://ucanr.edu/sites/TSWVfieldriskindex

University of Georgia. 2018. Peanut Rx Disease Risk Index. University of Georgia College of Agriculture and Environmental Sciences. https://peanuts. caes.uga.edu/content/dam/caes-subsite/peanuts/docs/2018/2018-Peanut-RXDisease-Risk-Index.pdf

Wells, M. L., Culbreath, A. K., Csinos, A. S., and Todd, J. W. 2002a. Effects of a plant activator and insecticides on tobacco thrips (Thysanoptera: Thripidae) feeding, behavior, and survival. J. Agric. Urban Entomol. 19:117-120.

Wells, M. L., Culbreath, A. K., and Todd, J. W. 2002b. The effect of applications of acibenzolar-S-methyl on Tomato spotted wilt virus and thrips in peanut. Peanut Sci. 29:136-141.

Whitfield, A. E., Ullman, D. E., and German, T. L. 2005. Tospovirus-thrips interactions. Annu. Rev. Phytopathol. 43:459-489. 